\title{
Screening for differentially expressed circRNA between Kashin-Beck disease and osteoarthritis patients based on circRNA chips
}

\section{Ying Wang}

the First Affiliated Hospital of Xi'an Jiao Tong University

\section{Cuiyan Wu}

Health Science Center of Xi'an Jiao Tong University

\section{Yanan Zhang}

Health Science Center of Xi'an Jiao Tong University

\section{Yimin Yang}

the First Affiliated Hospital of Xi'an Jiao Tong University

\section{Zhiwei Ren}

the First Affiliated Hospital of Xi'an Jiaotong University

Mikko J. Lammi

Umea Universitet

Xiong Guo ( $\sim$ guox@outlook.com )

Xi'an Jiaotong University

\section{Research article}

Keywords: osteoarthritis; Kashin-Beck disease; circRNAs expression profiles; biomarkers

Posted Date: September 23rd, 2019

DOI: https://doi.org/10.21203/rs.2.14818/v1

License: (c) (i) This work is licensed under a Creative Commons Attribution 4.0 International License.

Read Full License

Version of Record: A version of this preprint was published at Clinica Chimica Acta on February 1st, 2020. See the published version at https://doi.org/10.1016/j.cca.2019.10.026. 


\section{Abstract}

Objective: The purpose of this research was to explore differentially expressed circRNA between OA and $\mathrm{KBD}$ and the potential differential diagnosis biomarkers.

Methods: Total RNA was extracted from 5 pairs of KBD and OA knee joint cartilage specimens, and the expression of circRNAs was analyzed by Chip Scanning Analysis. The microarray data was verified by quantitative polymerase chain reaction (qRT-PCR) analysis. GO enrichment analysis and KEGG pathway were used to predict the functions of the differentially expressed circRNAs. A circRNA-miRNA network was constructed to predict targeting microRNAs of differentially expressed circRNA genes. On the basis of microarray, we expanded the sample size, peripheral blood samples from $25 \mathrm{KBD}$ patients and $25 \mathrm{OA}$ patients (five pairs of patients for chondrocyte microarray study included) were collected and qRT-PCR was performed for hsa_circRNA_0020014 verification. Diagnostic value was evaluated by the area under the receiver operator characteristic (ROC) curve.

Results: A total of 1627 circRNAs were differentially expressed between $O A$ and $K B D(P<0.05 ; 0.5<f$ old change>2), 1328 were up-regulated and 299 were down-regulated among them. Five differentially expressed genes associated with bone and joint disease were chosen for further qRT-PCR validation. After obtaining the parental genes of them, functional annotations were performed on the top ten enrichment GO items and KEGG pathways. The difference in expression profile of hsa_circRNA_0020014 was confirmed by qRT-PCR at articular cartilage level, and its circRNA-miRNA regulation network was set up. The ROC curve demonstrated that hsa_circ_0020014_CBC1 in peripheral blood could distinguish patients with KBD and OA (area under the curve: 0.6415, P< 0.01). The hsa_circ_0020014_CBC1 may be a potential biomarker for differential diagnosis between KBD and OA patients

Conclusion: Our results suggested that the expression profiles of circRNA were significantly different between OA and KBD. hsa_circRNA_0020014 is a potential biomarker for the differential diagnosis between these two diseases.

\section{Background}

CircRNAs belong to the non-coding RNA family and are highly expressed in eukaryotic cells. First found in plants and confirmed to encode a sub-virus [1], they are characterized by being single-stranded, covalent, and closed loops [2]. Later, circRNA was found to be highly expressed in mammalian brain tissues [3]. It has been demonstrated that the circRNA transcripts of sex-determining region $\mathrm{Y}$ (SRY) and circular RNA CDR1as [4] can be used as miRNA sponges [5]. Studies have shown that circRNA is widely involved in gene expression regulation, and has been proved to play an important role in the occurrence and development of nervous system diseases [6]. Due to their high degree of conservation [7], stablility [8] and accessible characteristics, the circRNAs could serve as biomarkers for diagnostics of clinical disease, such as gastric cancer [9], hepatocellular carcinoma [10] and oesophageal cancer [11]. The 
hsa_circ_0005836 was found as a biomarker and therapeutic target of active pulmonary tuberculosis [12].

Osteoarthritis $(\mathrm{OA})$ is a joint disease characterized by cartilage degeneration and secondary subchondral bone thickening, osteophyte formation and synovitis, which eventually leads to joint stiffness, pain, swelling and functional loss $[13,14]$. Due to tissue wear and chondrocyte death, cytokines and growth factors lead to increased catabolism and abnormal differentiation in OA, leading to degradation of extracellular matrix (ECM) [15].

Kashin-Beck disease (KBD) is a unique osteoarthropathy in China, diagnosed based on the national KBD diagnostic criteria (WS/T 207-2010). The disease is mainly distributed in the low-selenium areas of the soil from the northeast to the southwest of China. At present, there are more than 2.5 million current KBD patients and about 30 million residents at high risk [16]. Similar to OA, such as chondrocyte degeneration, ECM degradation and other basic pathological features were displayed $[17,18]$, although specific differences between $\mathrm{OA}$ and KBD chondrocyte gene expressions have been revealed [19]. In contrast to $\mathrm{OA}$, which commonly appears in middle-aged and elderly people, the onset of KBD occurs mostly in 3-12 years old children. The main clinical manifestations of KBD were enlarged finger joints, short fingers, curvature of distal fingers and short stature [17]. Until now, the diagnosis of KBD has been based on clinical features and typical imaging characteristics of metaphyseal bone, typical manifestations are irregular sclerosis and the appearance of calcification. KBD may be classified as early, stage I, II and III, based on the "Diagnosis Criteria of KBD in China (WS/T207-2010). The above methods are not sensitive enough to the early stage diagnosis of the disease, and it is difficult to intervene early in the late performance such as short stature and joint deformation.

At present, the generally accepted pathogenic theories of environmental factors concerning the pathogenesis of KBD include environmental selenium deficiency, high levels of humic acid in drinking water and contamination of grains by mycotoxins [20]. The exact pathologic mechanism of KBD is not yet fully understood. Because of the similar clinical manifestations between KBD and OA, KBD is known as an endemic OA. In recent years, circRNA has been proved to be a new biomarker in human peripheral blood [21] for diagnosis of diseases, such as coronary artery disease, OA and rheumatoid arthritis (RA) [22-24]. Until now, there have been no identification and characterization of circRNAs for OA and KBD in bone or cartilage tissue as well as in peripheral blood. Therefore, we focused on articular chondrocytes of patients with $\mathrm{KBD}$ and $\mathrm{OA}$, to explore the genetic features that could be associated with early diagnosis.

\section{Methods}

\subsection{The definitions of $K B D$ and $O A$ and collection of chondrocyte samples}

In our research, adult patients with KBD were diagnosed in accordance with WS/T 207-2010 criteria, OA patients were diagnosed based on the Western Ontario and McMaster Universities OA Index of stiffness 
and pain [25]. Patients with genetic bone disease and RA were excluded. In this research, a total of 10 participants were selected and matched according to gender and age and were divided into two groups (5 patients with KBD and 5 patients with $\mathrm{OA}$ ). Samples were selected randomly from outpatients and inpatients at The First Affiliated Hospital of Xi'an Jiaotong University, and from the KBD- endemic area Linyou County (Baoji, China) from April 2016 to October 2017. The study was approved by the Human Ethics Committee of Xi'an Jiaotong University, project approval number (2016-28), and all subjects signed informed consent. KBD and OA chondrocyte samples were taken from the same anatomical region of the femoral condyle of the knee joints. The chondrocyte specimens were obtained and cut part rapidly, and then frozen in liquid nitrogen and immediately stored at $-80^{\circ} \mathrm{C}$ until RNA extraction.

\subsection{Collection of peripheral blood samples}

Total of $3 \mathrm{ml}$ peripheral blood was drawn from each donor before breakfast by peripheral venipuncture and gathered in ethylene diamine tetra acetic acid (EDTA) anticoagulant vacutainers, and kept in the $80^{\circ} \mathrm{C}$ degree refrigerator until used for RNA isolation.

\subsection{Isolation and extraction of RNA}

First, total RNA was collected from the joint cartilage samples of five OA patients and five KBD patients using the TRIzol reagent Invitrogen (Invitrogen Corporation, Gaithersburg, MD, USA) and purification of the extracted RNA was performed use the mirVana miRNA Isolation Kit (Ambion, Austin, TX, USA) following the manufacturer's recommendation. Then the RNA was dissolved in RNase-free water. The yield and purity were monitored using a NanoDrop 2000 instrument (Thermo Scientific, Waltham, MA, USA). Samples were analyzed in a $1 \%$ formaldehyde denaturing agarose gel to confirm RNA integrity. Complementary DNA (cDNA) was generated by reverse transcription using Prime Script RT Reagent Kit (Takara Bio, Nojihigashi, Kusatsu, Japan) according to the manufacturer's instructions. Then, we expanded the sample size, total RNA was extracted from the peripheral blood samples of twenty-five patients with $\mathrm{OA}$ and twenty-five patients with KBD (Five pairs of patients for chondrocyte microarray study included).

\subsection{CircRNA microarray analysis}

The circRNA microarray analysis was summarized, normalized and quality control using GeneSpring software v13.0 (Agilent Technologies). The differentially expressed circRNAs in OA and KBD chondrocytes were identified through fold change, hierarchical clustering was used to display differentially expressed circRNAs between them. The limit values are as follows: fold change for upregulated circRNAs was $\geq 2$ and for down-regulated ones $\leq 0.5$ and $\mathrm{P}<0.05$. 


\section{5 qRT-PCR validation of microarray data}

While writing this paper, we have consulted a lot of relevant literature. Matta C et al [26] published a review article in 2014 showing that the calcium signaling pathway may be related to OA, while the target gene PDE1C of hsa_circ_0134111_CBC1 participates in the same pathway. Wang $L$ et al [27] reported in 2015 that the PRKCH gene is associated with RA, systemic lupus erythematosus, ankylosing spondylitis and OA, our research found that the target gene of hsa_circ_0032131_CBC1 is PRKCH. Sun HY et al reported in 2017 that the MAPK signaling pathway is related to OA, and the target gene dusp 5 of hsacirc-0020014-cbc1 is involved in the MAPK signaling pathway [28]. In 2018, Hu J et al [29] found that the development of microRNA-17-5p was closely related to the development of $O A$, and the prediction of microRNA-17-5p could be combined with hsa_circ_0094742_CBC1. Fang $Y$ et al reported in 2019 [30] that OA may be associated with protein digestion and absorption, while col3a1, the target gene of hsacirc-0057421- $\mathrm{CBC1}$, is involved in protein digestion and absorption. The above literature is the theoretical basis for us to select the five differentially expressed genes for further qRT-PCR verification. The selected circRNAs were analyzed using the ABI PRISM 7500 Sequence Detection System (Applied Biosystems, Life Technologies, Waltham, MA, USA). The primers used in our study are listed in Table 1.

\section{6 qRT-PCR verification of related circRNAs in peripheral blood samples}

To further validate the results of microarray analysis, the significant differentially expressed gene was chosen as target genes for qRTPCR. Total RNA in peripheral blood samples were reverse transcribed into cDNA using superscript II reverse transcriptase (invitrogen; Thermo Fisher Scientific, Inc.) according to the product's technical guidelines. The selected circRNA was analyzed using the Applied Biosystems ${ }^{\circledR}$ Quant Studio $^{\text {TM }} 7$ Flex Real-Time PCR System (Invitrogen ${ }^{T M}$ Life Technologies $^{\text {TM }}$, Carlsbad, CA). The primer specificity was confirmed at the melting curve stage. The PCR results were analyzed using Quant Studio ${ }^{\text {TM }}$ Real-Time PCR Software Vesersion1.1 (Invitrogen ${ }^{\mathrm{TM}}$ Life Technologies $^{\mathrm{TM}}$, Carlsbad, CA).

\subsection{Prediction of circRNA-targeting miRNAs}

As a miRNA sponge, circRNA exerts transcriptional control through competitive endogenous RNAs (CeRNAs) mechanisms. We used miRanda miRNA prediction software based on the miRanda3.3 software to explore functional annotations of the identified circRNA and predict circRNA/miRNA interactions. The selected circRNA-miRNA pairs were chosen to construct the network with the open source bioinformatics software Cytoscape. Then a circRNA-miRNA interaction network has been created.

\subsection{Data analysis}


First, raw data extraction from scanned images by Genepix Pro 6.0 software (Axon; Molecular Devices, LLC, Sunnyvale, CA, USA). Next, the original data is normalized and processed using the R software package. Finally, exclude low-intensity substances. The fold change of each cirRNA was calculated and the difference between $\mathrm{OA}$ and KBD patients was compared. The limit values are as follows: $\mathrm{P}<0.05 a n d$ fold change $\geq 2$ and $\leq 0.5$, the differentially expressed circRNAs were arranged according to the $P$-value and fold change.

\section{Results}

\subsection{Differential expression profiling of circRNAs in knee joint chondrocytes of KBD and OA}

Differentially expressed circRNAs were distinguished between OA and KBD chondrocyte samples according to the statistical analysis of the microarray data. A total of 1627 circular RNA expressions were significantly different, containing 1328 up-regulated circRNAs and 299 down-regulated circRNAs, the top 10 most significant circRNAs are shown in Table 2. According to the refseq database annotation of circRNAs, the genomic origin of human circRNAs includes introns, exons, intergenic regions, untranslated regions, antisense regions of known transcripts and unmarked regions of the genome [31]. Hierarchical clustering was performed to reflect all differentially expressed circRNA expression profiles. The microarray expression profiles of circRNAs are represented in figure 1 . The top 20 differentially expressed circRNAs in the KBD group and the OA group are shown in figure 1A. The scatter plot represents the

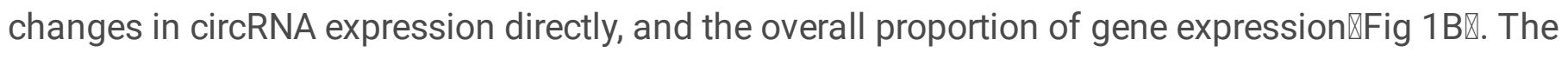
volcano plot reflects the number, significance and reliability of different genes. The closer each point in the graph is to the upper left corner and the upper right corner, the more significant the difference is (Fig 1C). Among the up-regulated circRNAs, 1037 were exonic, 2 intronic, 289 were in the other genomic regions, while the down-regulated circRNAs consisted of 254 exonic, 1 intronic circRNA, and 44 in the other genomic regions (Figure 2).

Figure 1. Identification of differentially expressed circRNAs in KBD and OA patients.

Fig.1A. The cluster graph of the top 20 circRNAs Cluster diagram directly reflect the similarity between groups, each column represents a sample, each line represents a circRNA, with red indicating a relatively high level of expression and the green part denoting a relatively low level of expression. The color bar in the upper left corner represents a comparison table with numbers and colors, the top sample tree represents the clustering relationships of similarity between samples. Fig.1B. The scatter plot expresses the changes in circRNA expression, the horizontal ordinate represents the average expression value of OA samples, the vertical axis represents the average expression value of KBD samples. Both abscissa and ordinate use the value transformed by log2. The up-regulated genes labeled red, down-regulated genes labeled green and non-significantly different genes labeled black. Fig.1C. The volcano plot intuitively reflects the number, significance and reliability of differentially expressed genes. The horizontal ordinate 
is - $\log 10$ (p-values) and the vertical axis is log2 (fold change). The closer each point in the graph is to the upper left corner and the upper right corner, the more significant the difference is.

Figure 2. Classification of differentially expressed genes according to genome origin.

\section{2. qRT-PCR validation of differentially expressed circRNAs between $\mathrm{OA}$ and KBD chondrocyte samples}

The chosen 5 differentially expressed circRNAs for further validation by qRT-PCR. There were one upregulated circRNA (hsa_circ_0057421_CBC1) and four down-regulated circRNAs

(hsa_circ_0020014_CBC1, hsa_circ_0032131_CBC1, hsa_circ_0094742_CBC1, hsa_circ_0134111_CBC1)

for validation among the five pairs of cartilage samples (five patients with primary $O A$ and five with endemic KBD), hsa_circ_20014_CBC1 has been shown to be the most reliable in terms of reliability in microarrays and RT-PCR (Figure 3).

Figure 3. The consistency of qRT-PCR and chip analysis of 5 differentially expressed circRNA molecules. From left to right represent Hsa_circ_0020014_CBC1, hsa_circ_0032131_CBC1, hsa_circ_0057421_CBC1, hsa_circ_0094742_CBC1, hsa_circ_0134111_CBC1.

\subsection{Bioinformatics analysis of differentially expressed circRNAs between OA and KBD}

In our research, we used the KOBAS (KEGG Orthology Based Annotation System) to analyze the differentially expressed circRNAs and their parental genes, $\mathrm{GO}$ analyses were performed on three different aspects: cellular component (CC), biological process (BP), and molecular function (MF). The top 10 enriched GO entries are displayed in Figure 4, the most enriched CC terms were related to "organelle part (GO: 0044422)", "centriole (GO: 0005814)" and "microtubule cytoskeleton (GO:0015630)". The most enriched and meaningful BP related to "cell projection organization (GO: 0030030)", "triglyceride mobilization (GO: 0006642)", and "inositol biosynthetic process (GO: 0006021)". Regarding MF terms, the most enriched terms were related to "inositol monophosphate phosphatase activity (GO: 0052834)", "ATP binding (GO: 0005524)", and "adenyl ribonucleotide binding (GO: 0032559)". In addition, we also conducted KEGG pathway analysis (Figure 5), and the top 10 pathways are shown. The most enriched and meaningful pathways from the BioCyc database are related to "D-myo-inositol $(1,4,5)$-trisphosphate degradation", "ascorbate recycling (cytosolic)" and "mRNA capping", "phosphatidylinositol signaling system", "RNA degradation" and "RNA transport" (from the KEGG pathway database). The most enriched and meaningful pathways from the PANTHER database are related to the "PDGF signaling pathway", "inflammation mediated by chemokine and cytokine signaling pathway", "endothelin signaling pathway", and the "p53 pathway". From the Reactome database, the most meaningful and enriched pathways were "Rev-mediated nuclear export of HIV RNA", "regulation of HSF1-mediated heat shock response" and 
"interactions of Rev with host cellular proteins". The KEGG disease pathway (Figure 6) gives us the following enlightenment, the most enriched and meaningful disease pathways were related to "vascular diseases, short rib-polydactyly syndrome, distal hereditary motor neuropathies" (from KEGG-disease database). "epidermolysis bullosa, generalized atrophic benign, chondrodysplasia with joint dislocations (GPAPP type), and Parkinson's disease" from Online Mendelian Inheritance in Man (OMIM) database.

Figure 4. Functional annotations for the parental genes of circRNAs in the $O A$ and KBD groups, (GO.p_value.top, the top10 from all terms in the three categories of $B P, C C$, and MF).

Figure 5. Functional annotations for the parental genes of circRNAs in the OA and KBD groups, and KEGG analysis of the pathways of the differential circRNA parental genes from four different biological pathway databases (the Kyoto Encyclopedia of Genes and Genomes, BioCyc, PANTHER and Reactome). The figure shows only the top 10 enrichment KEGG pathways. The enrichment factor is the ratio of the number of genes in the $\mathrm{GO}$ entry to the total number of genes in the $\mathrm{GO}$ entry. The larger the enrichment factors the greater the enrichment.

Figure 6. Disease annotations for the parental genes of circRNAs. Analysis of the disease pathways of the differential circRNA parental genes from three different disease pathways databases (NHGRI_GWAS_Catalog, OMIM, KEGG_DISEASE).

\subsection{Related target miRNAs and the miRNA/circRNA interaction network}

Considering microarray analysis and verification results, hsa_circRNA_0020014 was expressed significantly differentially in chondrocytes between OA patients and KBD patients and closely related to bone and joint diseases. To explore target miRNAs that may be associated with OA and KBD, the validated hsa_circRNA_0020014 was selected for further analysis and the miRNA/circRNA interaction network was predicted by miRNA targeting prediction software. We ranked the top 10 from low to high according to the tot energy (table 3 ), and the circRNA/microRNA regulatory network diagram of hsa_circ_0020014_CBC1 was drawn on this basis (Figure 7).

Figure 7. CircRNA-miRNA regulation network constructed and visualized by Target Scan and miRanda.

Network diagram shows the interaction based on the predicted target genes of hsa_circRNA_0020014 targeting miRNAs.

\subsection{Further validation in peripheral blood of OA and KBD patients}


On the basis of circRNA microarray, we expanded the sample size, confirmed the results by qRT-PCR and ROC (receiver operator characteristic) curve analysis. Peripheral blood samples from 25 KBD patients and 25 OA patients (five pairs of patients for chondrocyte microarray study included) were collected and qRTPCR was performed for hsa_circRNA_0020014 verification. The ROC curve demonstrated that hsa_circ_0020014_CBC1 in peripheral blood could distinguish patients with KBD and OA (area under the curve: 0.6415, P<0.01). The hsa_circ_0020014_CBC1 may be a potential biomarker for differential diagnosis between KBD and OA patients (Figure 8). When hsa_circ_0020014_CBC1 = 9.478 CT, the Yoden index reaches a maximum of 0.30 , the sensitivity and specificity were 0.90 and 0.30 , respectively.

Figure 8. ROC curve of hsa_circ_0020014_CBC1, hsa_circ_0032131_CBC1 in peripheral blood of KBD patients and OA patients (two ROC graphs will be combined into one picture later).

\section{Discussion}

Protein-coding genes and their transcription in eukaryotic cells have been extensively studied [32]. The reality is that a large number of sequences in the human genome do not encode proteins, and non-coding RNA accounts for almost $95 \%$ of the total RNA transcribed from the eukaryotic genome, including circular RNA [33]. There are two main types of RNA molecules: endogenous RNA molecules formed by nonlinear reverse splicing and intron-derived cyclic RNA molecules [33]. In the 1970s, circular RNA was first discovered in RNA viruses [34, 35]. CircRNA was also found in the transcripts of human cells in 1993, but at that time, it was considered to be a low-abundance RNA molecule formed by mis-splicing of exons during transcription [36]. With the rapid development of RNA sequencing and bioinformatics, circRNAs are not a rare phenomenon, but present widely in the cytoplasm of eukaryotic cells and play an important role in regulating gene expression at the post-transcriptional level. Studies have shown that circRNAs acts as a microRNA sponge that competitively inhibits miRNA activity and thereby regulating target genes of miRNA. Research has shown that circRNAs are involved in the initiation and development of many kinds of diseases. For example, due to the deficiency in the "sponge" effect of CIRS-7, miRNA-7 is upregulated, lead to down-regulating Alzheimer's disease-relevant target genes [6]. Some scholars have reported that circRNAs play an important role in the progression of cardiovascular diseases, such as hsa_circ_0124644, which may be associated with coronary artery disease [22]. And many studies have reported that circRNAs are closely related to a variety of tumors. In one study, Weihai Liu et al [37] noted that hsa_circRNA_103801 and hsa_circRNA_104980 can be used as biomarkers for the diagnosis and treatment of osteosarcoma. Research on circRNAs relating to osteoarticular diseases mainly includes the following results. Some studies have systematically explored the potential functions of non-coding RNAs in osteoclast genesis by microarray technology, and constructed co-expression networks of IncRNAmRNA and circRNA-miRNA [38].

In another study, circRNA was shown to potentially affect the development and progression of RA and play an important role in the regulation of related gene expression (24). Liu Qiang et al explored the function of human cartilage degeneration mechanical stress-related circRNA (circRNAs-MSR) and concluded that inhibition of circRNAs-MSR can limit the degradation of the ECM of chondrocytes, 
therefore, circRNAs-MSR may be a potential target for the treatment of osteoarthritis [39]. However, as a prevalent degenerative joint disease, the role of circRNAs in the pathogenesis of OA is not very clearly. $A$ recent study indicated that hsa-circ- 0045714 inhibits the progression of OA by promoting aggregation of type II collagen and proteoglycan expression and chondrocyte proliferation [40]. For the special endemic $\mathrm{OA}(\mathrm{KBD})$ in China, there has been no report related to circRNA until now.

In our study, we found a large number of differentially expressed circRNAs in OA and KBD cartilage specimens. The microarray analysis between KBD and osteoarthritis chondrocytes was conducted to analyse the differentially expressed circRNAs. A total of 1627 circRNAs were differentially expressed, include 1328 upregulated circRNAs and 299 downregulated circRNAs. We use qRT-PCR to detect the expression levels of five selected circRNAs, the results were consistent with the results of the analysis of the microarray analysis data, demonstrating the reliability of the spectrogram. GO and KEGG pathway analyses were used for functional annotation of differentially expressed circRNA genes. We found that these genes are involved in RNA transport, RNA degradation, and the p53 pathway. The most enriched and meaningful disease pathways were related to "vascular diseases, short rib-polydactyly syndrome, distal hereditary motor neuropathies" (from KEGG-disease database). "generalized atrophic benign, chondrodysplasia with joint dislocations (GPAPP type), and Parkinson's disease" (from OMIM database).

In addition, the most significantly differentially expressed and confirmed circRNA (hsa_circRNA_0020014) was further explored and revealed the potential molecular mechanisms of regulation by circRNAs in joint disease pathogenesis. we expanded the number of samples of the subjects, $25 \mathrm{KBD}$ patients and $25 \mathrm{OA}$ patients were selected and divided into two groups according to the age and gender matching principle, peripheral blood samples were collected and RNAs were extracted for qRT-PCR verification. Peripheral blood verification results were consistent with chondrocyte verification results, and hsa_circ_0020014_CBC1 was significantly different between OA and KBD. Moreover, ROC curve was used to evaluate the diagnostic value and reliability, the levels of hsa_circ_0020014_CBC1 in peripheral blood appeared potential diagnostic value between KBD and OA, and a high ROC AUC value $(0.6415, P<0.01)$ compared with traditional bone metabolism indicators, it has great potential as a diagnostic biomarker. Furthermore, research on the function of hsa_circ_0020014_CBC1 can improve the understanding of the mechanism of these two diseases occurrence and development.

According to the sponge theory, we predicted the potential target miRNAs and constructed a circRNAmiRNA interaction regulation network. The predicted microRNAs were selected according to tot energy using Cytoscape software, and the smaller the energy was, the tighter interaction between circRNAs and miRNAs. Top ten predicted miRNAs of hsa_circRNA_0020014 were identified in the networks. Therefore, they are theoretically related to osteoarthrosis and require further study. Two of the top 10 predicted miRNAs have been reported in a recent study, hsa-circ-0061012 was up-regulated in psoriasis lesions compared to normal healthy skin tissue [41]. Some scholars reported that miR-6743-5p acts as an oncomiRNA in glioma by targeting GRIM-19 and STAT3 (39). And miR-6743-5p expression is downregulated in patients with lymph node metastasis in oesophageal cancer [42]. There is no definitive report on $\mathrm{OA}$ and $\mathrm{KBD}$ in these predicted targeted microRNAs. The gene symbol corresponding to 
hsa_circRNA_0020014 is dual-specificity phosphatase 5 (DUSP-5), studies have shown that in in DUSP5overexpressing mice, the severity of arthritis, as shown by clinical arthritis scores and tissue inflammation and cartilage damage was attenuated [43]. Pramanik noted that mutations in dusp- 5 and snrk -1 have been found in tissues of patients with vascular disorder [44]. Wickramasekera NT and his team have drawn conclusions in a study showing that DUSP- 5 appears to regulate the signalling of pressuredependent myogenic cerebral arterial constriction, it is essential for maintaining the blood supply to the brain [45].

\section{Conclusion}

We used high-throughput information analysis to explore the potential biological function of circRNAs in human primary $\mathrm{OA}$ and endemic $\mathrm{OA}(\mathrm{KBD})$ in China for the first time. In compliance with WHO requirements for minimally invasive biomarkers with clinical significance we enlarged the sample size and extracted RNA from peripheral blood for further validation of previous research results. From this, we can draw the following conclusion: hsa_circ_0020014_CBC1 can be detected from peripheral blood at a relatively low cost, fast, and minimally invasive. Therefore, hsa_circ_0020014_CBC1 can be used as a biomarker for differential diagnosis between OA and KBD.

\section{Declarations}

Acknowledgements

We are grateful to the medical staff of the Department of Orthopaedics, the First Affiliated Hospital of Xi'an Jiaotong University for providing clinical material, and thank Health Science Center of Xi'an Jiaotong University.

\section{Availability of data and materials}

The data and materials provided are authentic and reliable.

Authors' contributions

All authors participated in the drafting of the manuscript and critical revisions of important intellectual content, and all authors approved the publication of the paper.

Study design and quality control: Ying Wang, Xiong Guo, Mikko J. Lammi

Data collection: Cuiyan Wu, Yanan Zhang

Data analysis and annotation: Ying Wang, Yimin Yang, Zhiwei Ren

Writing-original draft: Yimin Yang

Ethics approval and consent to participate

Page $11 / 25$ 
The Human Ethics Committee of Xi'an Jiaotong University (Xi'an, China) approved the beginning of this study. The study was conducted according to the approved regulations, and all subjects provided informed consent.

Competing interests

All authors declared no conflict of interest.

\section{Reference}

1.Sanger HL, Klotz G, Riesner D, Gross HJ, Kleinschmidt AK. Viroids are single-stranded covalently closed circular RNA molecules existing as highly base-paired rod-like structures. Proceedings of the National Academy of Sciences of the United States of America. 1976;73(11):3852-6. doi: 10.1073/pnas.73.11.3852. PubMed PMID: 1069269; PubMed Central PMCID: PMC431239.

2.Jeck WR, Sharpless NE. Detecting and characterizing circular RNAs. Nature biotechnology. 2014;32(5):453-61. doi: 10.1038/nbt.2890. PubMed PMID: 24811520; PubMed Central PMCID: PMC4121655.

3.Hansen TB, Wiklund ED, Bramsen JB, Villadsen SB, Statham AL, Clark SJ, et al. miRNA-dependent gene silencing involving Ago2-mediated cleavage of a circular antisense RNA. The EMBO journal. 2011;30(21):4414-22. doi: 10.1038/emboj.2011.359. PubMed PMID: 21964070; PubMed Central PMCID: PMC3230379.

4.Hansen TB, Jensen TI, Clausen BH, Bramsen JB, Finsen B, Damgaard CK, et al. Natural RNA circles function as efficient microRNA sponges. Nature. 2013;495(7441):384-8. doi: 10.1038/nature11993. PubMed PMID: 23446346.

5.Capel B, Swain A, Nicolis S, Hacker A, Walter M, Koopman P, et al. Circular transcripts of the testisdetermining gene Sry in adult mouse testis. Cell. 1993;73(5):1019-30. PubMed PMID: 7684656.

6.Lukiw WJ. Circular RNA (circRNA) in Alzheimer's disease (AD). Frontiers in genetics. 2013;4:307. doi: 10.3389/fgene.2013.00307. PubMed PMID: 24427167; PubMed Central PMCID: PMC3875874.

7.Salzman J, Gawad C, Wang PL, Lacayo N, Brown PO. Circular RNAs are the predominant transcript isoform from hundreds of human genes in diverse cell types. PloS one. 2012;7(2):e30733. doi:

10.1371/journal.pone.0030733. PubMed PMID: 22319583; PubMed Central PMCID: PMC3270023.

8.Suzuki H, Tsukahara T. A view of pre-mRNA splicing from RNase R resistant RNAs. International journal of molecular sciences. 2014;15(6):9331-42. doi: 10.3390/ijms15069331. PubMed PMID: 24865493; PubMed Central PMCID: PMC4100097.

9.Li P, Chen S, Chen H, Mo X, Li T, Shao Y, et al. Using circular RNA as a novel type of biomarker in the screening of gastric cancer. Clinica chimica acta; international journal of clinical chemistry. 
2015;444:132-6. doi: 10.1016/j.cca.2015.02.018. PubMed PMID: 25689795.

10.Qin M, Liu G, Huo X, Tao X, Sun X, Ge Z, et al. Hsa_circ_0001649: A circular RNA and potential novel biomarker for hepatocellular carcinoma. Cancer biomarkers: section A of Disease markers. 2016;16(1):161-9. doi: 10.3233/CBM-150552. PubMed PMID: 26600397.

11.Li F, Zhang L, Li W, Deng J, Zheng J, An M, et al. Circular RNA ITCH has inhibitory effect on ESCC by suppressing the Wnt/beta-catenin pathway. Oncotarget. 2015;6(8):6001-13. doi:

10.18632/oncotarget.3469. PubMed PMID: 25749389; PubMed Central PMCID: PMC4467417.

12.Zhuang ZG, Zhang JA, Luo HL, Liu GB, Lu YB, Ge NH, et al. The circular RNA of peripheral blood mononuclear cells: Hsa_circ_0005836 as a new diagnostic biomarker and therapeutic target of active pulmonary tuberculosis. Molecular immunology. 2017;90:264-72. doi: 10.1016/j.molimm.2017.08.008. PubMed PMID: 28846924.

13. Hayami T, Pickarski M, Zhuo Y, Wesolowski GA, Rodan GA, Duong LT. Characterization of articular cartilage and subchondral bone changes in the rat anterior cruciate ligament transection and meniscectomized models of osteoarthritis. Bone. 2006;38(2):234-43. doi: 10.1016/j.bone.2005.08.007. PubMed PMID: 16185945.

14.Dieppe P, Kirwan J. The localization of osteoarthritis. British journal of rheumatology. 1994;33(3):2013. PubMed PMID: 8156279.

15.Kuhn K, D'Lima DD, Hashimoto S, Lotz M. Cell death in cartilage. Osteoarthritis and cartilage. 2004;12(1):1-16. PubMed PMID: 14697678.

16.Fang H, Guo X, Farooq U, Xia C, Dong R. Development and validation of a quality of life instrument for Kashin-Beck disease: an endemic osteoarthritis in China. Osteoarthritis and cartilage. 2012;20(7):630-7. doi: 10.1016/j.joca.2012.03.004. PubMed PMID: 22430051.

17.Wang SJ, Guo X, Zuo H, Zhang YG, Xu P, Ping ZG, et al. Chondrocyte apoptosis and expression of Bcl2, Bax, Fas, and iNOS in articular cartilage in patients with Kashin-Beck disease. The Journal of rheumatology. 2006;33(3):615-9. PubMed PMID: 16511931.

18.Guo X, Ma WJ, Zhang F, Ren FL, Qu CJ, Lammi MJ. Recent advances in the research of an endemic osteochondropathy in China: Kashin-Beck disease. Osteoarthritis and cartilage. 2014;22(11):1774-83. doi: 10.1016/j.joca.2014.07.023. PubMed PMID: 25106677.

19.Duan C, Guo X, Zhang XD, Yu HJ, Yan H, Gao Y, et al. Comparative analysis of gene expression profiles between primary knee osteoarthritis and an osteoarthritis endemic to Northwestern China, Kashin-Beck disease. Arthritis and rheumatism. 2010;62(3):771-80. doi: 10.1002/art.27282. PubMed PMID: 20131229. 
20.Zou K, Liu G, Wu T, Du L. Selenium for preventing Kashin-Beck osteoarthropathy in children: a metaanalysis. Osteoarthritis and cartilage. 2009;17(2):144-51. doi: 10.1016/j.joca.2008.06.011. PubMed PMID: 18693119.

21.Memczak S, Papavasileiou P, Peters O, Rajewsky N. Identification and Characterization of Circular RNAs As a New Class of Putative Biomarkers in Human Blood. PloS one. 2015;10(10):e0141214. doi: 10.1371/journal.pone.0141214. PubMed PMID: 26485708; PubMed Central PMCID: PMC4617279.

22.Shan K, Liu C, Liu BH, Chen X, Dong R, Liu X, et al. Circular Noncoding RNA HIPK3 Mediates Retinal Vascular Dysfunction in Diabetes Mellitus. Circulation. 2017;136(17):1629-42. doi:

10.1161/CIRCULATIONAHA.117.029004. PubMed PMID: 28860123.

23.Ning Y, Wang X, Wang S, Guo X. Comparative analysis of signaling pathways in peripheral blood from patients with Kashin-Beck disease and osteoarthritis. Experimental and therapeutic medicine.

2016;12(6):4077-84. doi: 10.3892/etm.2016.3879. PubMed PMID: 28101186; PubMed Central PMCID: PMC5228111.

24.Zheng F, Yu X, Huang J, Dai Y. Circular RNA expression profiles of peripheral blood mononuclear cells in rheumatoid arthritis patients, based on microarray chip technology. Molecular medicine reports. 2017;16(6):8029-36. doi: 10.3892/mmr.2017.7638. PubMed PMID: 28983619; PubMed Central PMCID: PMC5779885.

25.Ehrich EW, Davies GM, Watson DJ, Bolognese JA, Seidenberg BC, Bellamy N. Minimal perceptible clinical improvement with the Western Ontario and McMaster Universities osteoarthritis index questionnaire and global assessments in patients with osteoarthritis. The Journal of rheumatology. 2000;27(11):2635-41. PubMed PMID: 11093446.

26. Matta C, Mobasheri A. Regulation of chondrogenesis by protein kinase C: Emerging new roles in calcium signalling. Cellular signalling. 2014;26(5):979-1000. doi: 10.1016/j.cellsig.2014.01.011. PubMed PMID: 24440668.

27.Wang L, Wu LF, Lu X, Mo XB, Tang ZX, Lei SF, et al. Integrated Analyses of Gene Expression Profiles Digs out Common Markers for Rheumatic Diseases. PloS one. 2015;10(9):e0137522. doi:

10.1371/journal.pone.0137522. PubMed PMID: 26352601; PubMed Central PMCID: PMC4564267.

28.Sun HY, Hu KZ, Yin ZS. Inhibition of the p38-MAPK signaling pathway suppresses the apoptosis and expression of proinflammatory cytokines in human osteoarthritis chondrocytes. Cytokine. 2017;90:13543. doi: 10.1016/j.cyto.2016.11.002. PubMed PMID: 27907835.

29.Hu J, Wang Z, Shan Y, Pan Y, Ma J, Jia L. Long non-coding RNA HOTAIR promotes osteoarthritis progression via miR-17-5p/FUT2/beta-catenin axis. Cell death \& disease. 2018;9(7):711. doi: 10.1038/s41419-018-0746-z. PubMed PMID: 29907764; PubMed Central PMCID: PMC6003907. 
30.Fang Y, Wang P, Xia L, Bai S, Shen Y, Li Q, et al. Aberrantly hydroxymethylated differentially expressed genes and the associated protein pathways in osteoarthritis. PeerJ. 2019;7:e6425. doi:

10.7717/peerj.6425. PubMed PMID: 30828485; PubMed Central PMCID: PMC6394344.

31.Memczak S, Jens M, Elefsinioti A, Torti F, Krueger J, Rybak A, et al. Circular RNAs are a large class of animal RNAs with regulatory potency. Nature. 2013;495(7441):333-8. doi: 10.1038/nature11928. PubMed PMID: 23446348.

32.Esteller M. Non-coding RNAs in human disease. Nature reviews Genetics. 2011;12(12):861-74. doi: 10.1038/nrg3074. PubMed PMID: 22094949.

33.Jeck WR, Sorrentino JA, Wang K, Slevin MK, Burd CE, Liu J, et al. Circular RNAs are abundant, conserved, and associated with ALU repeats. Rna. 2013;19(2):141-57. doi: 10.1261/rna.035667.112. PubMed PMID: 23249747; PubMed Central PMCID: PMC3543092.

34.Wilusz JE, Sharp PA. Molecular biology. A circuitous route to noncoding RNA. Science. 2013;340(6131):440-1. doi: 10.1126/science.1238522. PubMed PMID: 23620042; PubMed Central PMCID: PMC4063205.

35.Wu Q, Wang Y, Cao M, Pantaleo V, Burgyan J, Li WX, et al. Homology-independent discovery of replicating pathogenic circular RNAs by deep sequencing and a new computational algorithm. Proceedings of the National Academy of Sciences of the United States of America. 2012;109(10):393843. doi: 10.1073/pnas.1117815109. PubMed PMID: 22345560; PubMed Central PMCID: PMC3309787.

36. Cocquerelle C, Mascrez B, Hetuin D, Bailleul B. Mis-splicing yields circular RNA molecules. FASEB journal: official publication of the Federation of American Societies for Experimental Biology. 1993;7(1):155-60. doi: 10.1096/fasebj.7.1.7678559. PubMed PMID: 7678559.

37.Liu W, Zhang J, Zou C, Xie X, Wang Y, Wang B, et al. Microarray Expression Profile and Functional Analysis of Circular RNAs in Osteosarcoma. Cellular physiology and biochemistry: international journal of experimental cellular physiology, biochemistry, and pharmacology. 2017;43(3):969-85. doi: 10.1159/000481650. PubMed PMID: 28957794.

38.Dou C, Cao Z, Yang B, Ding N, Hou T, Luo F, et al. Changing expression profiles of IncRNAs, mRNAs, circRNAs and miRNAs during osteoclastogenesis. Scientific reports. 2016;6:21499. doi: 10.1038/srep21499. PubMed PMID: 26856880; PubMed Central PMCID: PMC4746671.

39.Liu Q, Zhang X, Hu X, Yuan L, Cheng J, Jiang Y, et al. Emerging Roles of circRNA Related to the Mechanical Stress in Human Cartilage Degradation of Osteoarthritis. Molecular therapy Nucleic acids. 2017;7:223-30. doi: 10.1016/j.omtn.2017.04.004. PubMed PMID: 28624198; PubMed Central PMCID: PMC5415239. 
40.Li BF, Zhang Y, Xiao J, Wang F, Li M, Guo XZ, et al. Hsa_circ_0045714 regulates chondrocyte proliferation, apoptosis and extracellular matrix synthesis by promoting the expression of miR-193b target gene IGF1R. Human cell. 2017;30(4):311-8. doi: 10.1007/s13577-017-0177-7. PubMed PMID: 28795385.

41.Qiao M, Ding J, Yan J, Li R, Jiao J, Sun Q. Circular RNA Expression Profile and Analysis of Their Potential Function in Psoriasis. Cellular physiology and biochemistry: international journal of experimental cellular physiology, biochemistry, and pharmacology. 2018;50(1):15-27. doi: 10.1159/000493952. PubMed PMID: 30278433.

42.Cao F, Zhang Q, Chen W, Zheng F, Ran Q, He Y, et al. miR-6743-5p, as a direct upstream regulator of GRIM-19, enhances proliferation and suppresses apoptosis in glioma cells. Bioscience reports. 2017;37(6). doi: 10.1042/BSR20171038. PubMed PMID: 29074558; PubMed Central PMCID: PMC5725612.

43.Moon SJ, Lim MA, Park JS, Byun JK, Kim SM, Park MK, et al. Dual-specificity phosphatase 5 attenuates autoimmune arthritis in mice via reciprocal regulation of the Th17/Treg cell balance and inhibition of osteoclastogenesis. Arthritis \& rheumatology. 2014;66(11):3083-95. doi: 10.1002/art.38787. PubMed PMID: 25047518.

44.Pramanik K, Chun CZ, Garnaas MK, Samant GV, Li K, Horswill MA, et al. Dusp-5 and Snrk-1 coordinately function during vascular development and disease. Blood. 2009;113(5):1184-91. doi: 10.1182/blood-2008-06-162180. PubMed PMID: 18927432; PubMed Central PMCID: PMC2635084.

45.Wickramasekera NT, Gebremedhin D, Carver KA, Vakeel P, Ramchandran R, Schuett A, et al. Role of dual-specificity protein phosphatase- 5 in modulating the myogenic response in rat cerebral arteries. Journal of applied physiology. 2013;114(2):252-61. doi: 10.1152/japplphysiol.01026.2011. PubMed PMID: 23172031; PubMed Central PMCID: PMC3544499.

\section{Tables}

Table 1: Primers used in our research:

\begin{tabular}{ll}
\hline gene & Sequences (from 5’ to 3’) \\
\hline hsa_circ_0020014_CBC1 & F CGTGTGAATGTGAAGAAAAGCAG \\
& R CGAGGAACTCGCACTTGGAT \\
hsa_circ_0032131_CBC1 & F TACCTGGCTCCATGAAGATGC \\
& R CCTCCTTGCACATTCCGAAG \\
hsa_circ_0134111_CBC1 & F CAGGAGAACAGCAACAGAATGG \\
& R CTGCTTCCTTCTTGGCCTTC \\
hsa_circ_0057421_CBC1 & F AAAATTCGGACCTGTTGGACC \\
& R TCTTTCACCTCTGTTACCTCG \\
hsa_circ_0094742_CBC1 & F GAGGAATCAGCCTGCTGAATATAG \\
& R TCATTATTGATCTGCTTTAGAACCC \\
\hline
\end{tabular}

Table 2: The top 10 most significant differentially expressed circRNAs selected according to p-value and fold change: 


\begin{tabular}{llllll}
\hline circRNA & Regulation & Chromosome & geneSymbol & P-value & FC(abs) \\
\hline hsa_circ_0139061_CBC1 & up & chr9 & PTPRD & 0.20 & 6.99 \\
hsa_circ_0139055_CBC1 & up & chr9 & PTPRD & 0.27 & 6.04 \\
hsa_circ_0139074_CBC1 & up & chr9 & PTPRD & 0.36 & 5.89 \\
hsa_circ_0060652_CBC1 & up & chr20 & PREX1 & 0.28 & 5.73 \\
hsa_circ_0073466_CBC1 & up & chr5 & ERAP2 & 0.32 & 5.72 \\
hsa_circ_0114299_CBC1 & up & chr1 & TTLL7 & 0.29 & 5.60 \\
hsa_circ_0139723_CBC1 & up & Chr X & - & 0.34 & 5.49 \\
hsa_circ_0101493_CBC1 & up & chr14 & NOVA1 & 0.36 & 5.43 \\
hsa_circ_0139080_CBC1 & up & chr9 & PTPRD & 0.36 & 5.36 \\
hsa_circ_0139062_CBC1 & up & chr9 & PTPRD & 0.38 & 5.32 \\
hsa_circ_0005180_CBC1 & down & chr1 & FMN2 & 0.33 & 6.50 \\
hsa_circ_0017822_CBC1 & down & chr10 & FRMD4A & 0.31 & 5.97 \\
hsa-circRNA6227_CBC1 & down & chr5 & CDH6 & 0.24 & 5.78 \\
hsa_circ_0093155_CBC1 & down & chr10 & FRMD4A & 0.29 & 5.62 \\
hsa_circ_0055265_CBC1 & down & chr2 & ACTG2 & 0.36 & 5.61 \\
hsa_circ_0026352_CBC1 & down & chr12 & NR4A1 & 0.25 & 5.60 \\
hsa-circRNA1779-4_CBC1 & down & chr12 & NR4A1 & 0.25 & 5.37 \\
hsa_circ_0026351_CBC1 & down & chr12 & NR4A1 & 0.28 & 5.01 \\
hsa_circ_0093151_CBC1 & down & chr10 & FRMD4A & 0.29 & 4.74 \\
hsa-circRNA3424-4_CBC1 & down & chr17 & IGF2BP1 & 0.44 & 4.72 \\
\hline & & & & &
\end{tabular}

Table 3: Top 10 most likely miRNA targets of hsa_circRNA_0020014 predicted and selected based on tot energy.

\begin{tabular}{lll}
\hline MicroRNA ID & Score & Tot energy \\
\hline hsa-miR-4763-3p & 165 & -32.88 \\
hsa-miR-4268 & 170 & -31.98 \\
hsa-miR-6893-3p & 172 & -31.92 \\
hsa-miR-6769b-5p & 166 & -31.05 \\
hsa-miR-6882-3p & 164 & -30.66 \\
hsa-miR-6743-5p & 166 & -30.51 \\
hsa-miR-5088-5p & 168 & -30.21 \\
hsa-miR-4758-5p & 164 & -29.91 \\
hsa-miR-3972 & 168 & -29.44 \\
hsa-miR-6076 & 169 & -28.73 \\
\hline
\end{tabular}

Figures 


\section{A the top 20 upregulated circRNAs}
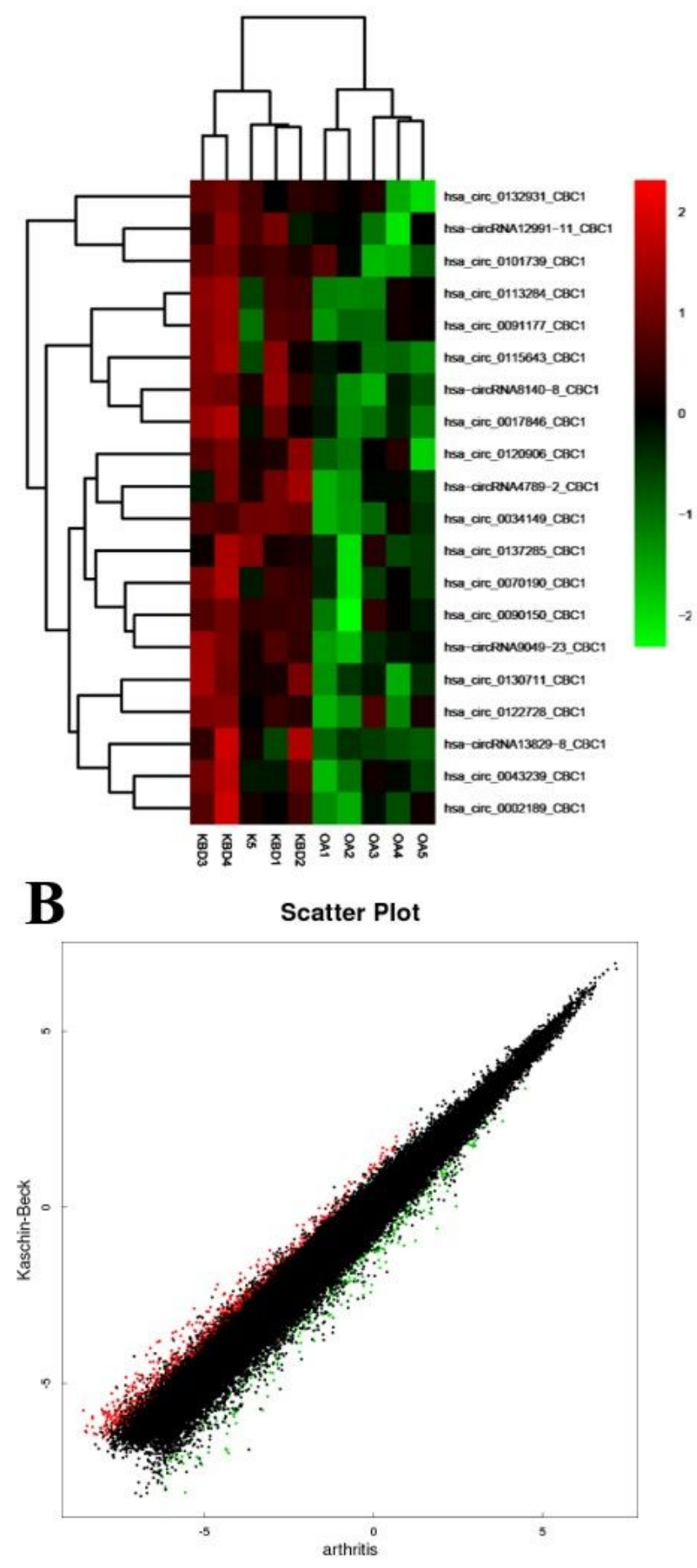

the top 20 down regulated circRNAs
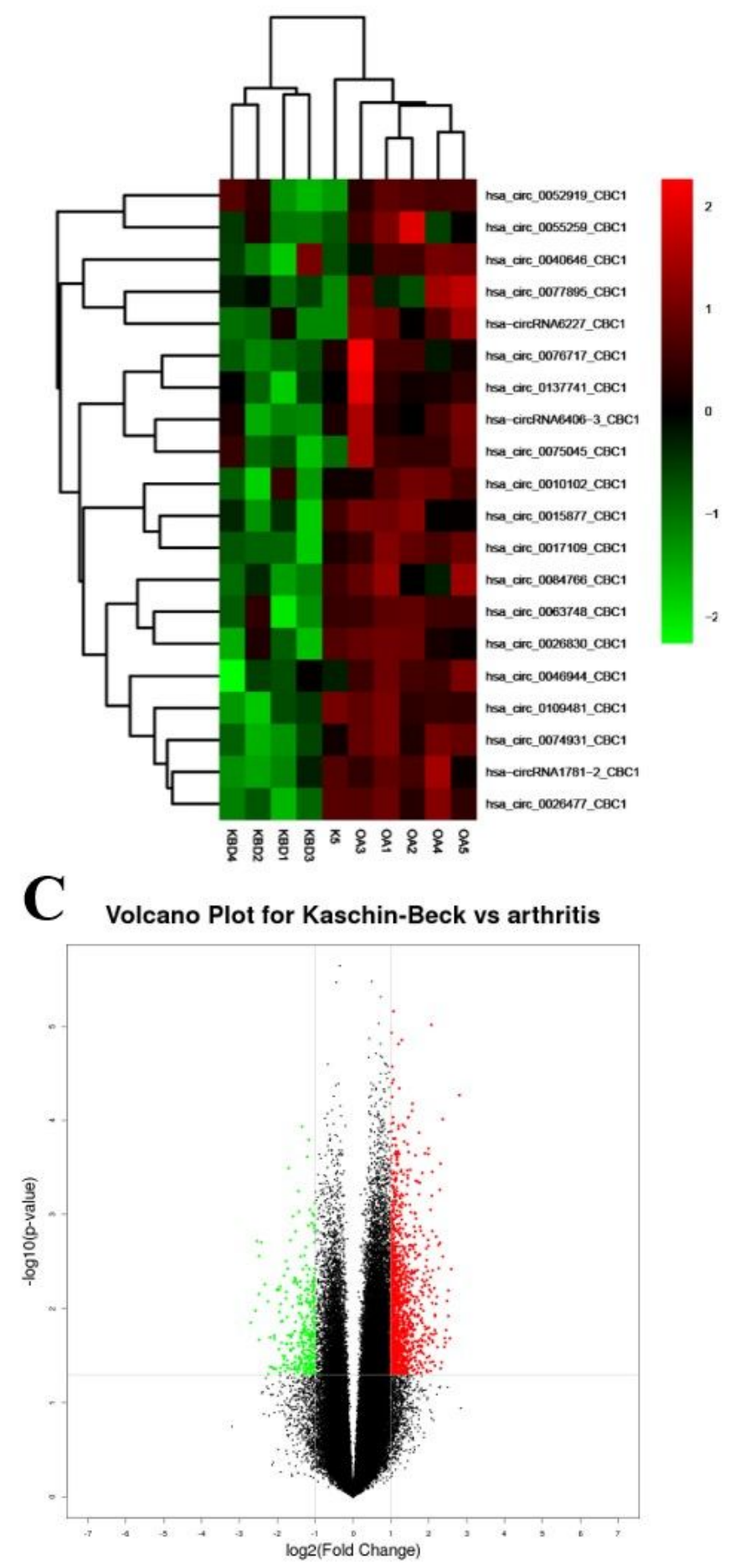

\section{Figure 1}

Identification of differentially expressed circRNAs in KBD and OA patients. Fig.1A. The cluster graph of the top 20 circRNAs Cluster diagram directly reflect the similarity between groups, each column represents a sample, each line represents a circRNA, with red indicating a relatively high level of expression and the green part denoting a relatively low level of expression. The color bar in the upper left corner represents a comparison table with numbers and colors, the top sample tree represents the 
clustering relationships of similarity between samples. Fig.1B. The scatter plot expresses the changes in circRNA expression, the horizontal ordinate represents the average expression value of OA samples, the vertical axis represents the average expression value of KBD samples. Both abscissa and ordinate use the value transformed by log2. The up-regulated genes labeled red, down-regulated genes labeled green and non-significantly different genes labeled black. Fig.1C. The volcano plot intuitively reflects the number, significance and reliability of differentially expressed genes. The horizontal ordinate is - $\log 10$ ( $p$ values) and the vertical axis is log2 (fold change). The closer each point in the graph is to the upper left corner and the upper right corner, the more significant the difference is.

\section{Up-regulation}

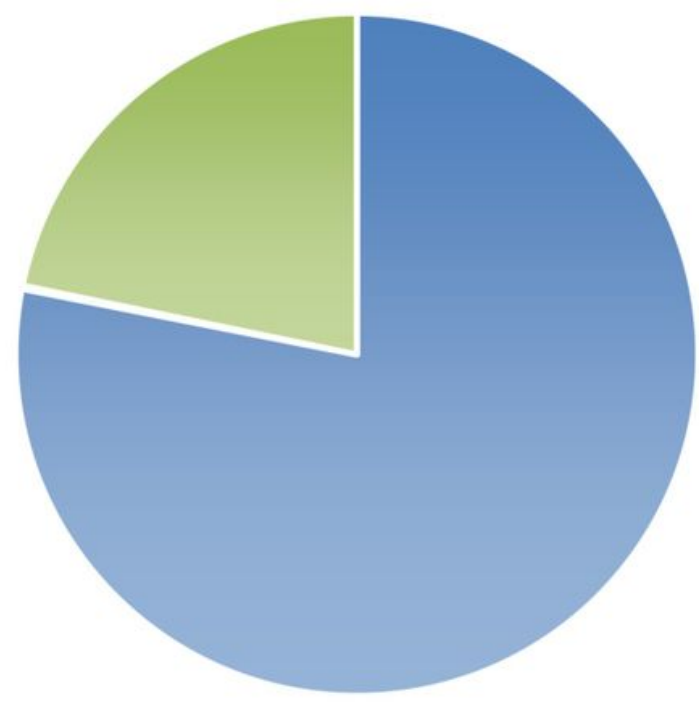

Total $=1328$
Down-regulation

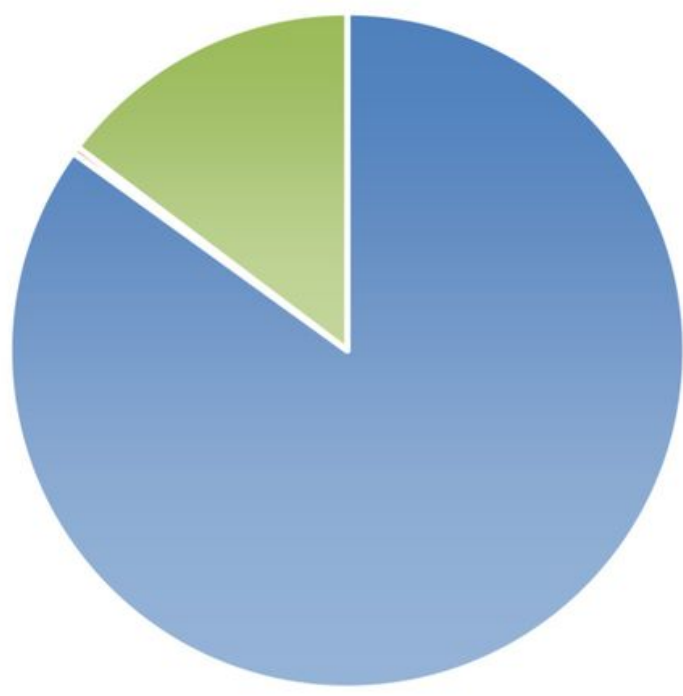

Total=299
= Exon

m intron

w other

\section{Figure 2}

Classification of differentially expressed genes according to genome origin. 


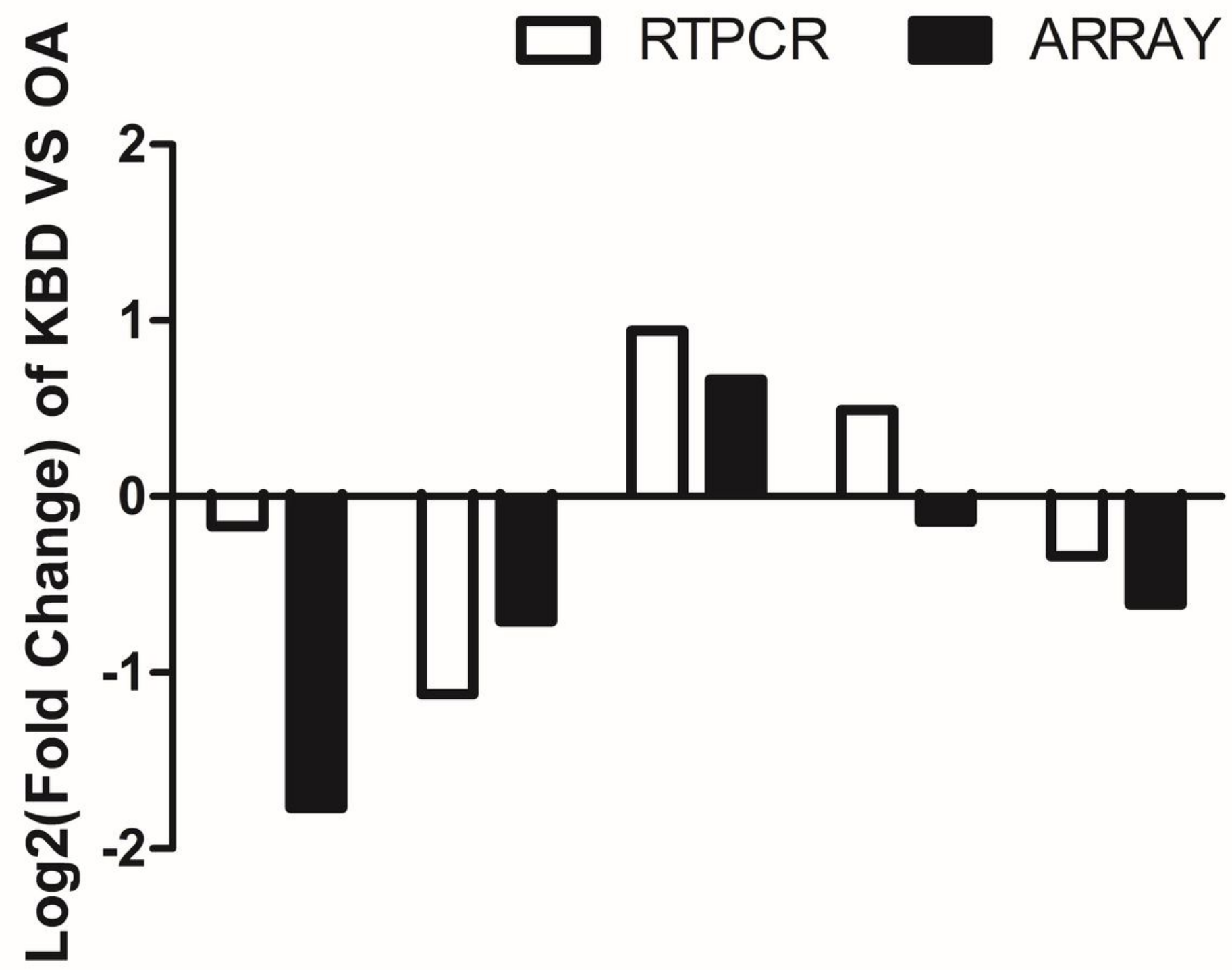

Figure 3

The consistency of qRT-PCR and chip analysis of 5 differentially expressed circRNA molecules. From left to right represent Hsa_circ_0020014_CBC1, hsa_circ_0032131_CBC1, hsa_circ_0057421_CBC1, hsa_circ_0094742_CBC1, hsa_circ_0134111_CBC1. 


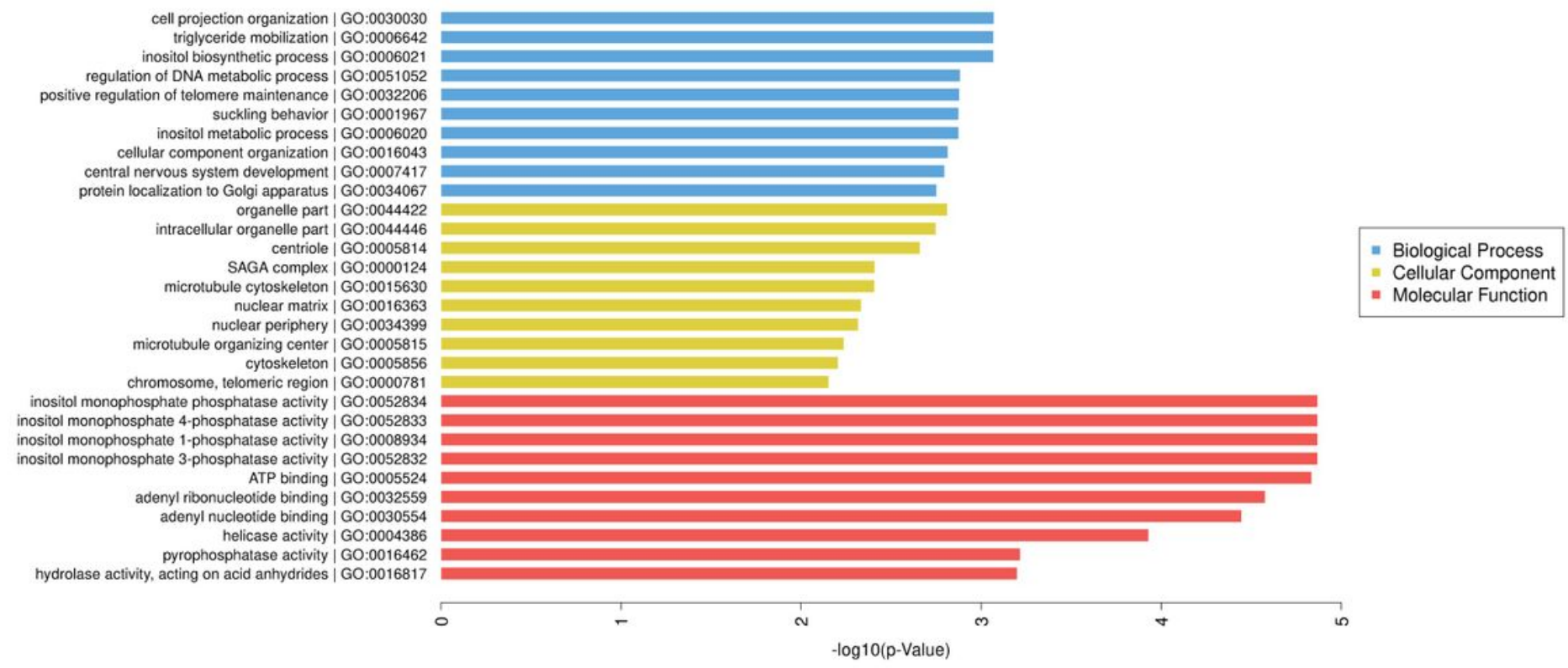

Figure 4

Functional annotations for the parental genes of circRNAs in the OA and KBD groups, (GO.p_value.top, the top10 from all terms in the three categories of BP, CC, and MF). 

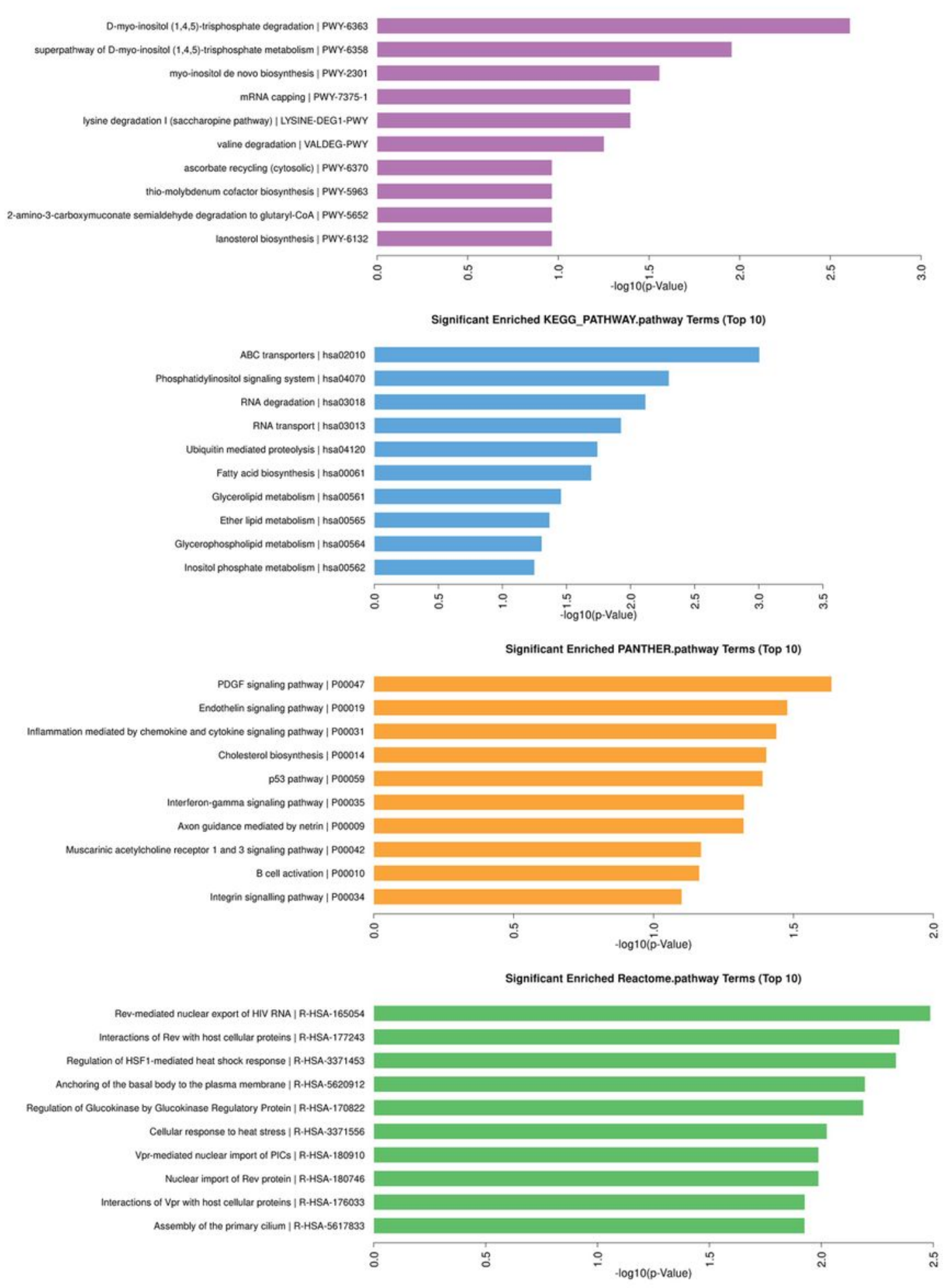

\section{Figure 5}

Functional annotations for the parental genes of circRNAs in the OA and KBD groups, and KEGG analysis of the pathways of the differential circRNA parental genes from four different biological pathway databases (the Kyoto Encyclopedia of Genes and Genomes, BioCyc, PANTHER and Reactome). The figure shows only the top 10 enrichment KEGG pathways. The enrichment factor is the ratio of the number of 
genes in the GO entry to the total number of genes in the GO entry. The larger the enrichment factors the greater the enrichment.

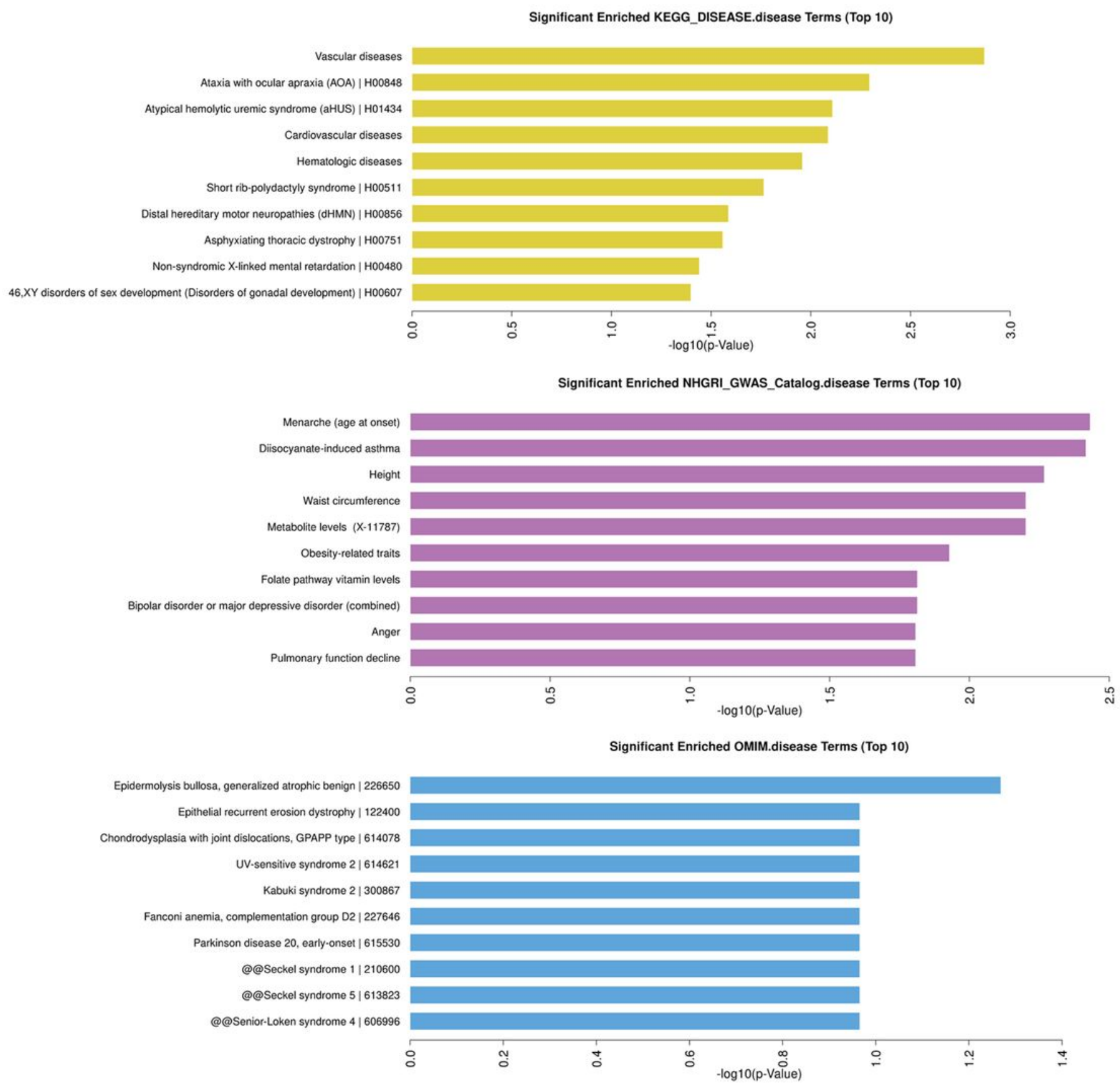

\section{Figure 6}

Disease annotations for the parental genes of circRNAs. Analysis of the disease pathways of the differential circRNA parental genes from three different disease pathways databases (NHGRI_GWAS_Catalog, OMIM, KEGG_DISEASE). 


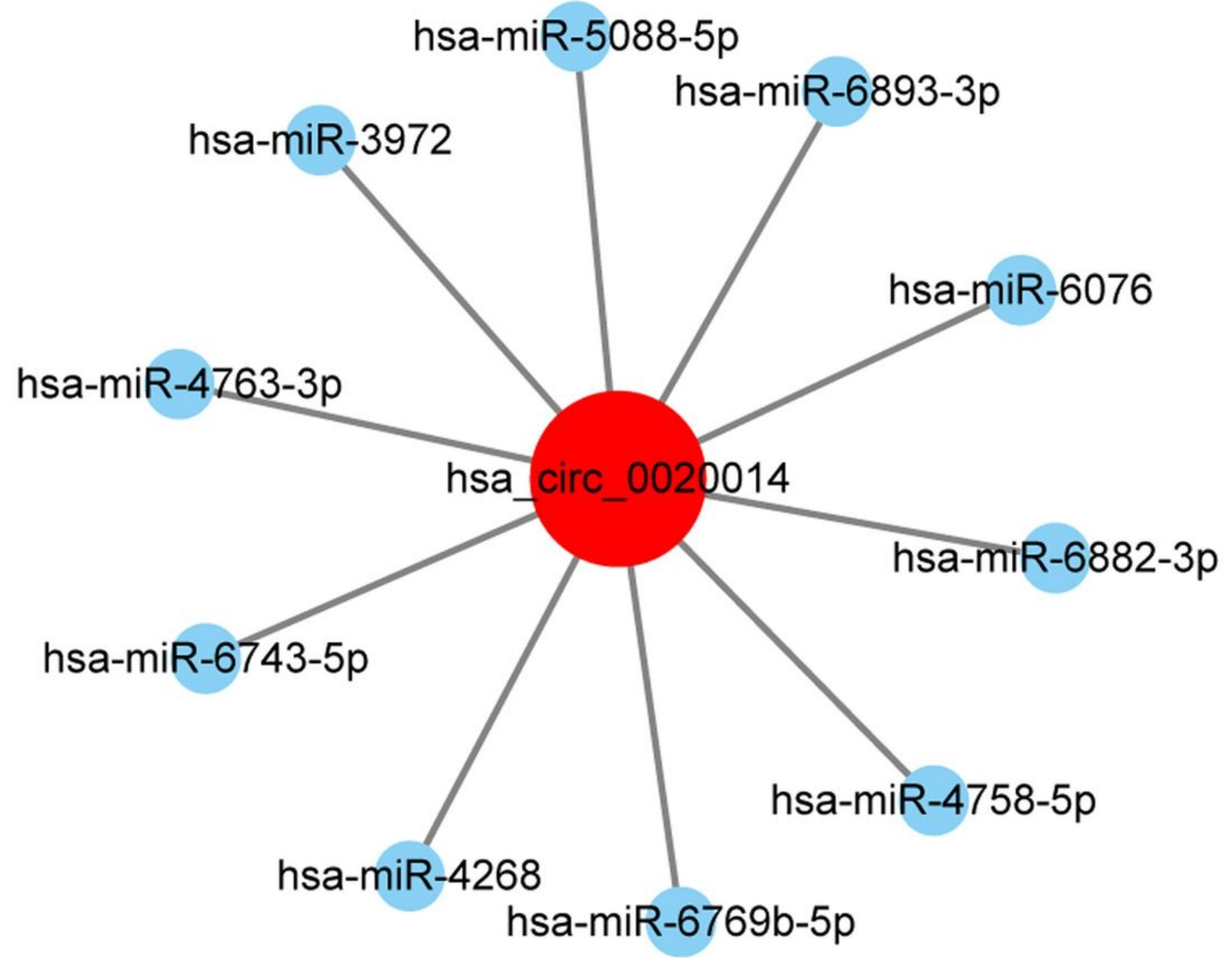

Figure 7

CircRNA-miRNA regulation network constructed and visualized by Target Scan and miRanda. Network diagram shows the interaction based on the predicted target genes of hsa_circRNA_0020014 targeting miRNAs. Table 3: Top 10 most likely miRNA targets of hsa_circRNA_0020014 predicted and selected based on tot energy. 
ROC curves from cross-validation

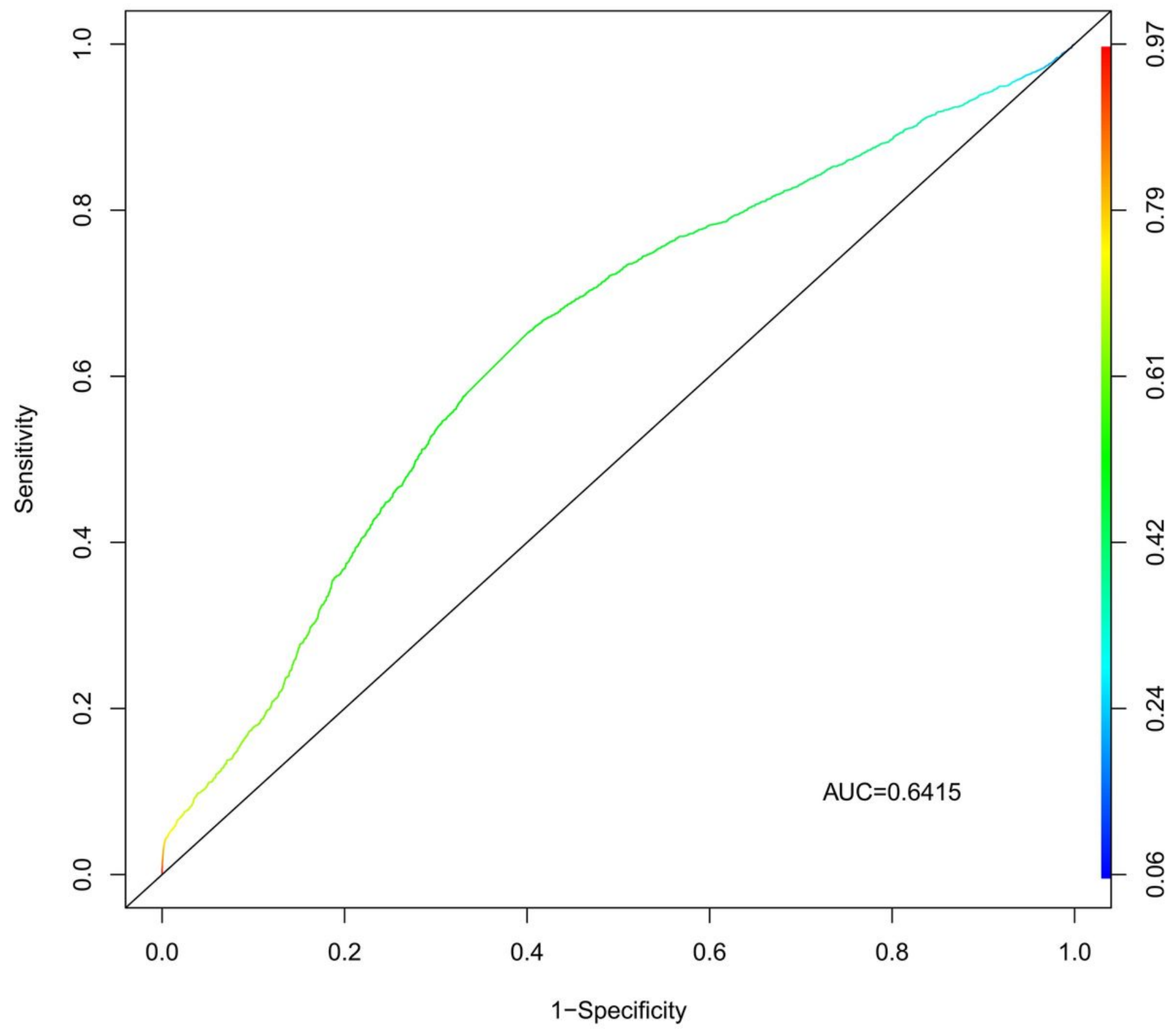

Figure 8

ROC curve of hsa_circ_0020014_CBC1, hsa_circ_0032131_CBC1 in peripheral blood of KBD patients and OA patients (two ROC graphs will be combined into one picture later). 